\title{
Inhaled Nitric Oxide: Effects on Cerebral Growth and Injury in a Baboon Model of Premature Delivery
}

\author{
SANDRA M. REES, EMILY J. CAMM, MICHELLE LOELIGER, SARAH CAIN, SANDRA DIENI, DONALD MCCURNIN, \\ PHILIP W. SHAUL, BRADLEY YODER, CATRIONA MCLEAN, AND TERRIE E. INDER
}

\begin{abstract}
Department of Anatomy and Cell Biology [S.M.R., E.J.C., M.L., S.C., S.D.], University of Melbourne, Parkville, Victoria, 3010, Australia; Department of Pediatrics, Neurology and Radiology [T.E.I.], St. Louis Children's Hospital, Washington University, St. Louis, Missouri 63130; Department of Pediatrics [D.M., P.W.S.], University of Texas Southwestern Medical Center, Dallas, Texas 75390; Southwest Foundation for Biomedical Research [B.Y.], San Antonio, Texas 78245; Anatomical Pathology Unit [C.M.], Alfred Hospital, Melbourne, Victoria, 3010, Australia
\end{abstract}

\begin{abstract}
Inhaled nitric oxide (iNO) enhances ventilation in very preterm infants, but the effects on the brain remain uncertain. We evaluated the impact of iNO on brain growth and cerebral injury in a premature baboon model. Baboons were delivered at $125 \mathrm{~d}$ of gestation (term $185 \mathrm{~d}$ of gestation) and ventilated for $14 \mathrm{~d}$ with either positive pressure ventilation (PPV) $(n=7)$ or PPV + iNO $(n=8)$. Brains were assessed histologically for parameters of development and injury. Compared with gestational controls $(n=7)$, brain and body weights were reduced but brain-to-body weight ratios were increased in all prematurely delivered (PD) animals; the surface folding index (SFI), was reduced in PPV but not PPV + iNO animals. Compared with controls, the brain damage index was increased $(p<0.05)$ in both cohorts of PD animals. There was no difference between ventilatory regimens, however, in $25 \%$ of animals with iNO therapy, there were organized hematomas in the subarachnoid space. Overall, iNO did not alter the extent of brain damage but did result in the presence of hematomas. These results do not confirm any protective or major injurious effect of nitric oxide therapy on the developing brain. (Pediatr Res 61: 552-558, 2007)
\end{abstract}

$\mathrm{N}^{\mathrm{e}}$ ew ventilatory strategies are one of the most important advances contributing to the increased survival rates for premature infants over the past few decades; however, the effects of these strategies on the developing brain are not completely understood. As the increased survival rates are associated with a high rate of poor neurodevelopmental outcome $(1,2)$, understanding the effects of these therapies on the developing brain is crucial.

iNO therapy improves oxygenation and reduces the need for extracorporeal membrane oxygenation in term infants (3). However, the benefits of this therapy in preterm infants with respiratory failure are controversial. Potential short-term benefits have been demonstrated including improved arterial oxygenation, a lowering of pulmonary vascular resistance, and a reduction in the duration of mechanical ventilation $(4,5)$.

Received July 18, 2006; accepted December 7, 2006.

Correspondence: Sandra Rees, Ph.D., Department of Anatomy and Cell Biology, University of Melbourne, 3010, Victoria, Australia; e-mail: s.rees@unimelb.edu.au

Supported by NIH grant R01 HL074942 and in part by grants from the National Institutes of Health (NHLBI grant HL-52636 and U01-HL063399) and the National Center for Research Resources to the Southwest National Primate Research Center (P51 RR013986).

DOI: $10.1203 / p d r .0 b 013 e 318045 b e 20$
Contrary to the original dogma that iNO was a specific pulmonary vasodilator without systemic effects, it now appears that iNO may have downstream effects (6).

Studies reporting on the impact of iNO on the risk of cerebral injury in the preterm infant are conflicting. With relation to periventricular leukomalacia (PVL), iNO therapy has been reported to decrease the severity (7), but not change the incidence (8). For intraventricular hemorrhage (IVH)/ intracranial hemorrhage $(\mathrm{ICH})$, iNO has been reported to decrease the incidence of severe hemorrhages $(5,7)$ and to cause no change in the overall incidence (7), no change in the severity (8), and no change in the progression of the hemorrhage (9). In the study of van Meurs et al. (8), infants that were $<1000 \mathrm{~g}$ had increased rates of severe $\mathrm{ICH}$, but the timing of the occurrence of the hemorrhages, whether before commencement of iNO therapy, was not certain. iNO has also been reported to cause no change in the evolution of brain injury (10).

In relation to later neurodevelopmental outcomes in preterm infants receiving iNO therapy, the data are also controversial with studies reporting no significant long-term effects (11) or an improved outcome at $2 \mathrm{y}$ of age (12). Because it remains contentious as to whether iNO or indeed exogenously produced NO $(13,14)$ is beneficial or injurious to the developing brain, further clinical and animal studies are required to establish the risk and benefits of iNO treatment.

To provide an insight into the relationships between neonatal respiratory regimens and brain development, a model has been developed in the immature baboon (Papio papio) (15). It has already been shown that iNO therapy improves pulmonary function and modifies lung growth in this model (16). The aim of the present study was to evaluate the effects of iNO therapy on brain growth and the pattern and severity of cerebral injury in baboons delivered at $67 \%$ of gestation, which is equivalent to approximately 26-28 wk in terms of human development. Animals were respirated with or without iNO in neonatal

\footnotetext{
Abbreviations: GFAP, glial fibrillary acidic protein; H\&E, hematoxylin and eosin; iNO, inhaled nitric oxide; IVH, intraventricular hemorrhage; MBP, myelin basic protein; PD, premature delivery; PPV, positive pressure ventilation; SFI, surface folding index
} 
intensive care for $14 \mathrm{~d}$, and an extensive morphometric study of the brain was undertaken. Indices of growth and development and cerebral damage were devised and compared with gestational controls.

\section{METHODS}

Animal studies were performed at the Southwest Foundation for Biomedical Research (SFBR) in San Antonio, TX; they conformed to American Association for Accreditation of Laboratory Animal Care guidelines.

Delivery and instrumentation. Pregnant baboon dams (Papio papio) with timed gestations underwent elective hysterotomy under general anesthesia. Study animals were delivered at $125 \pm 2 \mathrm{~d}$ (term $185 \mathrm{~d}$ ). At birth, animals were weighed, sedated, intubated, and treated with $4 \mathrm{~mL} / \mathrm{kg}$ surfactant (Survanta, courtesy Ross Laboratories, Columbus, $\mathrm{OH}$ ) before the initiation of ventilatory support.

Ventilatory management and NO replacement. The respiratory management of ventilated animals has been described in detail previously (16). One hour after delivery, iNO was administered to the experimental groups at a level of $5 \mathrm{ppm}$ with an INOVent and continued at this same level until the completion of the study at $14 \mathrm{~d}$ of age. To control for patent ductus arteriosus, all animals underwent surgical ductal ligation on d 6 of life regardless of whether there was previous spontaneous ductal closure (16). The study groups were PPV with ductal ligation (PPV) $(n=7)$; PPV with ductal ligation and nitric oxide therapy (PPV + iNO) $(n=8)$. Gestational control animals $(n=$ 7) were delivered at $140 \mathrm{~d}$ of gestation.

Histologic analysis. Brains were removed, weighed, immersed in $10 \%$ buffered formalin, and prepared for histologic analysis as previously described (15). The entire brain was sectioned into 5-mm blocks in the coronal plane and the right hemisphere processed to paraffin; $10(8-\mu \mathrm{m})$ sections were collected from the rostral surface of each block. A section from each block was stained with hematoxylin and eosin $(\mathrm{H} \& \mathrm{E})$ and assessed for gross morphologic changes. Hemorrhages were stained with Masson's trichrome (collagen), Van Gieson's stain (elastic fibers), reticulin (reticular fibers), and Perl's stain (hemosiderin). Deposition of hemosiderin suggest that the bleed had been present for $48 \mathrm{~h}$ or longer.

Immunohistochemistry for rabbit anti-glial fibrillary acidic protein (GFAP) (1:500, Sigma Chemical Co., St. Louis, MO) was used to identify astrocytes, rat anti-myelin basic protein (MBP) (1:100, Millipore, Billerica, MA) and biotinylated Lycopersicon esculentum (tomato) lectin (1:250; Sigma Chemical Co.) histochemistry was used to visualize macrophages/reactive microglia as previously described (15).

All analyses were performed on all brains in the study. Qualitative and quantitative measurements were made on coded slides.

Qualitative analysis. H\&E sections were assessed for hemorrhages (score: 1 , present; 0 , absent) and for the presence of lectin-positive reactive macrophages/microglial cells in gray and white matter.

Quantitative analysis. For each of the quantitative parameters, a mean was calculated for each animal and a mean of the means for the group.

Volumetric measurements. In H\&E stained sections, the cross-sectional area of the right hemisphere from each block was assessed using a digitizing tablet (Sigma Chemical Co. Scan Pro 4, Media Cybernetics). Volumes were then estimated using the Cavalieri principle; the total volumes of white matter, deep gray matter (basal ganglia, thalamus, and hippocampus), ventricle, and cortex were also assessed in this manner (17).

SFI. The Surface Folding Index (SFI), which gives an estimation of the expansion of the surface area relative to volume, was determined from H\&E-stained sections (15).

Percentage of white matter occupied by blood vessels. Point counting (18) was used to determine the density of blood vessel profiles in deep and subcortical white matter $(\times 660)$. A section was analyzed from each block, and a counting frame placed randomly in one region of deep and one region of subcortical white matter in each section; a mean value was calculated for each region. Assessment was performed in GFAP-immunoreactive (IR)stained sections as blood vessel profiles are clearly delineated.

Areal density of astrocytes. GFAP-IR cells were counted $(\times 660)$ in randomly selected areas $\left(0.2 \mathrm{~mm}^{2}\right)$ in each of the deep and subcortical white matter for each block, the cerebral cortex (three sites in blocks from frontal/ temporal, parietal/temporal, and occipital lobes), and the hippocampus (stratum radiatum in the CA1 region).

Areal density of oligodendrocytes. MBP-IR oligodendrocytes were counted $(\times 660)$ in a randomly selected area $\left(0.2 \mathrm{~mm}^{2}\right)$ in the deep and subcortical white matter in blocks from the frontal/temporal, parietal/ temporal, and occipital lobes and a mean calculated.
Myelination. In control brains, myelination (MBP-IR) was most advanced in the internal capsule with fibers extending into the subcortical white matter; this was given a score of 3 . The extent of myelination in the PD groups was scored against this standard in a section from the parietal/temporal region $(0$, no myelination; 1 , a few myelinated fibers; 2 , bundles of myelinated fibers; 3 , similar extent of myelination to controls).

Perivascular cuffing in the subcortical white matter. The extent of perivascular cuffing was assessed in $\mathrm{H} \& \mathrm{E}$-stained sections at each level and scored on a scale of 0 to 3 ( 0 , not observed; 1 , occasionally observed; 2 , moderate degree; 3 , considerable number of vessels with cuffing).

GFAP-IR radial glial fibers. Sections from the frontal/temporal, parietal/ temporal, and occipital regions were scored for the presence of radial glial fibers on a scale of 0 to 3 ( 0 , not observed; 1 , occasionally observed; 2 , moderate degree; 3 , considerable number of fibers).

Growth and development index. To assess cerebral development in PD animals compared with controls, a growth index was constructed including body and brain weights and SFI (17). Data points were ranked from heaviest (22 points) to lightest ( 1 point) for weights and highest ( 22 points) to lowest (1 point) for SFI. Scores were added together and a mean calculated for each group.

Brain damage index. To assess the extent of structural alterations in PD compared with control animals, a brain index (19) was devised that included semiquantitative estimations (the extent of myelination, perivascular cuffing, and the presence of GFAP-IR radial glial fibers) and quantitative estimations (areal density of astrocytes in the subcortical and deep white matter and the cortex). Data points were ranked from the most affected (22 points) to least affected ( 1 point). The brain index was determined by adding the scores from each category

Physiologic data. Physiologic data, including arterial blood gases $\left(\mathrm{PaO}_{2}\right.$; $\mathrm{PaCO}_{2} ; \mathrm{pH}, \mathrm{FiO}_{2}$ ); systolic, diastolic, and mean arterial blood pressure (MAP); and heart rate, were monitored throughout the experimental period. The maximum and minimum values were recorded every $6 \mathrm{~h}$ for the first $2 \mathrm{~d}$ and daily thereafter. The overall maximum, minimum, and flux values were calculated for each parameter over the 14-d ventilatory period.

Regression analysis. Linear regression analysis was carried out to determine whether there was a correlation between (a) physiologic variables and the brain damage index, (b) physiologic variables and quantitative parameters (astrocyte densities, volumetric measurements), and (c) quantitative parameters (astrocyte densities) and indices of brain growth and development. A probability of $p<0.05$ was considered to be significant.

Data analysis. The statistical significance of differences between ventilated and control groups was tested using one-way analysis of variance with post hoc analysis (Tukey's test) for histologic parameters and $t$ tests to compare between ventilated groups for physiologic parameters; a probability of $p<0.05$ was considered to be significant. Results are expressed as mean \pm SEM (weights and areas) and mean of means \pm SEM (histologic parameters)

\section{RESULTS}

The group characteristics and the clinical variables have been reported previously (16). Briefly, the birth weights (PPV + iNO $418 \pm 36 \mathrm{~g}$, PPV $405 \pm 26 \mathrm{~g})$, gestational age (PPV + iNO $125 \pm 1$, PPV $125 \pm 2 \mathrm{~d}$ ), and male-to-female ratio (PPV + iNO 7/1, PPV 5:2) for the groups were not different.

\section{Growth and Development}

Brain and body weights (Table 1). Body and brain weights were reduced in both groups of PD animals compared with age-matched gestational controls $(p<0.001)$. The brain-tobody weight ratio was significantly higher $(p<0.05)$ in both PPV-treated and PPV + iNO animals compared with controls (Table 1).

Volumetric measurements (Table 1). Compared with controls the total volume of the right hemisphere $(p<0.001)$ and the cortical $(p<0.001)$, white matter $(p<0.001)$ and deep gray matter $(p<0.01)$ volumes were reduced in both groups. There was no difference between groups in the ratios of white matter, cortical or deep gray matter volume/total hemispheric volume or white matter/cortical volume $(p>0.05)$. Although 
Table 1. Body and brain weights and cerebral volumetric measurements

\begin{tabular}{|c|c|c|c|}
\hline Parameter & $\begin{array}{l}\text { Control } \\
(n=7)\end{array}$ & $\begin{array}{c}\text { PPV } \\
(n=7)\end{array}$ & $\begin{array}{c}\mathrm{PPV}+\mathrm{iNO} \\
(n=8)\end{array}$ \\
\hline Body weight at PM, g & $543.0 \pm 14.4$ & $339.3 \pm 18.7 *$ & $350.4 \pm 8.4^{*}$ \\
\hline Total brain weight at PM, $\mathrm{g}$ & $60.0 \pm 1.6$ & $45.0 \pm 2.6^{*}$ & $44.8 \pm 1.0^{*}$ \\
\hline Volume of (right) cerebral hemisphere, $\mathrm{mm}^{3}$ & $20177 \pm 777$ & $13860 \pm 325^{*}$ & $14305 \pm 850^{*}$ \\
\hline Cortical volume, $\mathrm{mm}^{3}$ & $8858 \pm 425$ & $5730 \pm 138^{*}$ & $6136 \pm 435^{*}$ \\
\hline White matter volume, $\mathrm{mm}^{3}$ & $9136 \pm 300$ & $6627 \pm 158^{*}$ & $6726 \pm 400^{*}$ \\
\hline White matter/total hemispheric volume, $\%$ & $45.9 \pm 0.9$ & $48.5 \pm 0.5$ & $47.7 \pm 0.7$ \\
\hline Cortex/total hemispheric volume, $\%$ & $44.3 \pm 0.8$ & $41.9 \pm 0.6$ & $43.3 \pm 0.6$ \\
\hline Deep gray matter/total hemispheric volume, $\%$ & $10.1 \pm 0.7$ & $9.5 \pm 0.3$ & $9.0 \pm 0.7$ \\
\hline Ventricular/total hemispheric volume, $\%$ & $1.0 \pm 0.1$ & $1.3 \pm 0.1$ & $1.4 \pm 0.3$ \\
\hline White matter/cortical volume, $\%$ & $1.04 \pm 0.04$ & $1.16 \pm 0.03$ & $1.10 \pm 0.02$ \\
\hline
\end{tabular}

All volume measurements were made on the right hemisphere.

$* p<0.001$ vs control; $\dagger p<0.05$ vs control; $\$ p<0.01$ vs control.

ventricular volumes were not different between groups $(p>$ 0.05), two PPV + iNO animals (Table 3,\#1 and \#2) exhibited ventriculomegaly with ventricular/hemispheric volume ratios at least $100 \%$ higher than the control mean value. These animals also had the lowest total volumes and lowest cortical and white matter volumes (data not shown).

SFI (Table 2). The SFI of the cerebral hemispheres was reduced $(p<0.01)$ overall in PPV animals compared with controls; specifically, there were reductions in the parietal/ temporal $(p<0.01)$ and occipital $(p<0.05)$ lobes compared with controls. The total hemispheric SFI for PPV + iNO animals was not reduced compared with controls $(p>0.05)$, although there was a reduction $(p<0.05)$ in the parietal/ temporal region (Table 2).

Growth and development index. The score was lower $(p<$ 0.001 ) for both PPV and PPV + iNO animals compared with controls; there was no difference $(p>0.05)$ between PD groups (Fig. 1A).

\section{Cerebral Neuropathologies}

Qualitative assessment. Lectin-positive activated microglia/macrophages were observed infrequently within the hemispheres, and there was no greater incidence in either ventilated groups than in controls. In $25 \%$ of animals exposed to iNO (\#1, \#2, Table 3), there was evidence of recent past bleeding (Fig. 2A) and of organizing hematomas (Fig. 2B) within the subarachnoid space between the inferior and mid temporal gyri. These foci comprised lysed blood cells admixed with apoptotic bodies, histiocytes, macrophages (Fig.

Table 2. SFI

\begin{tabular}{lcll}
\hline \multicolumn{1}{c}{ Control } & \multicolumn{1}{c}{ PPV } \\
Parameter & $(n=7)$ & $\begin{array}{c}\text { PPV }+ \text { iNO } \\
(n=8)\end{array}$ \\
\hline Total hemisphere & $43.9 \pm 2.2$ & $36.3 \pm 0.6^{*}$ & $40.0 \pm 1.3$ \\
Frontal/temporal lobes & $34.9 \pm 2.6$ & $31.2 \pm 1.7$ & $32.0 \pm 2.3$ \\
Parietal/temporal lobes & $52.5 \pm 3.2$ & $41.1 \pm 2.2^{*}$ & $44.1 \pm 1.2 \dagger$ \\
Occipital lobe & $51.0 \pm 2.9$ & $38.5 \pm 3.4 \dagger$ & $46.0 \pm 3.4$ \\
\hline
\end{tabular}

SFI was derived from the right hemisphere; the values for each lobe are means \pm standard error of the mean (SEM); total hemisphere is the mean of means \pm SEM.

$* p<0.01$ vs control; $\dagger p<0.05$ vs control.
$2 C$ ), hemosiderin deposits (Fig. 2D), and some neutrophils. There was a fibroblastic reaction and reactivity of endothelial cells with adjacent meningeal cell hyperplasia. There were intact vessels nearby or within the area, but that there was no evidence of residual vessel wall encircling the hematoma. There was no evidence of collagen deposition suggesting organization of between 7 and $14 \mathrm{~d}$ duration. These animals also exhibited increased ventricular/hemispheric volume ratios.

In one PPV + iNO (\#6) and one PPV (\#4) animal, there was evidence of meningeal cell proliferation associated with a proliferation of small capillaries with a hyalinized subendothelial matrix (Fig. $2 E$ and $F$ ). Extravasated intact red cells were seen adjacent to this region; as there was no associated cellular reaction, their presence is difficult to interpret. The presence of a similar hyalinized matrix in animals with and without iNO therapy suggests that this finding is independent of iNO.

Quantitative assessment-Areal density of astrocytes (Table 3). Compared with controls (Fig. 3A), there was an increase $(p<0.05)$ in the overall areal density of astrocytes in the deep white matter in PPV + iNO animals (Fig. 3C) but not in PPV animals (Fig. 3B); no regional differences were observed. There was no overall difference in areal density of astrocytes in either ventilated group compared with controls in the subcortical white matter $(p>0.05)$; on a regional basis, there was an increase in the occipital lobe of PPV + iNO animals $\left(305 \pm 32\right.$ cells $/ \mathrm{mm}^{2}$, control versus $443 \pm 26$ cells $\left./ \mathrm{mm}^{2}, \mathrm{PPV} \pm \mathrm{iNO}, p<0.01\right)$. There was no overall increase in the areal density of astrocytes in the cerebral cortex
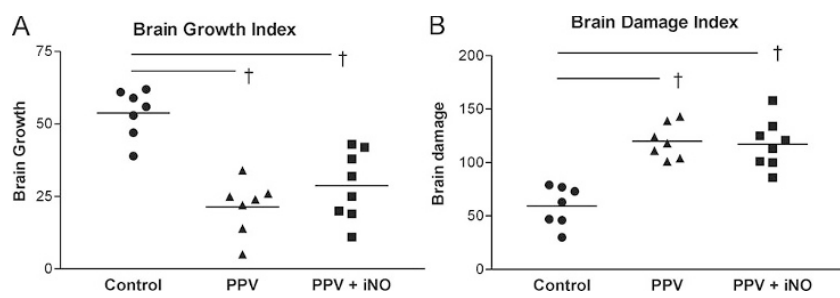

Figure 1. (A) The growth and development index scores were decreased $(\dagger p<$ 0.001) in both ventilated groups compared with controls. $(B)$ The brain damage index scores were increased $(\dagger p<0.001)$ in both ventilated groups (PPV, $n=7$; PPV $+\mathrm{iNO}, n=8)$ compared with controls $(n=7)$. 

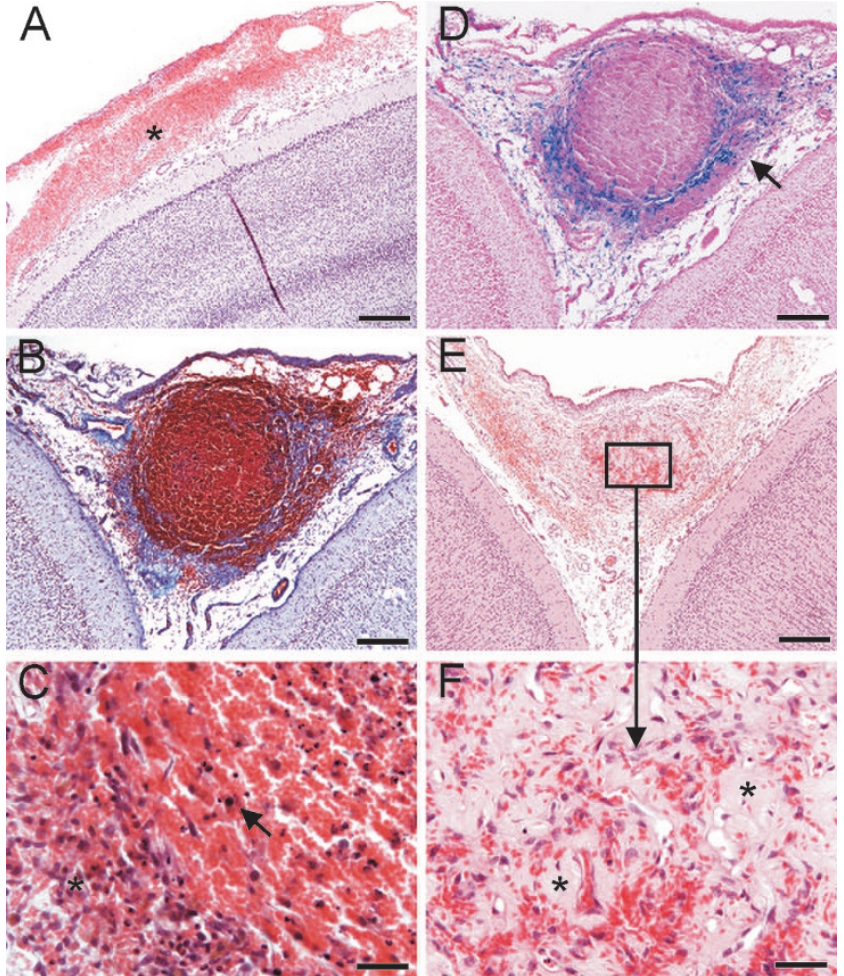

Figure 2. (A) Subarachnoid hemorrhage $(*)$ associated with hematoma stained with $\mathrm{H} \& \mathrm{E}$. (B) An organized hematoma in the subarachnoid space with proliferation of meningeal cells; Masson's trichrome stain. $(C)$ Higher magnification of the center of the hematoma shows apoptotic bodies (arrow), macrophages, and erythrocytes (*). (D) Perl's-stained hematoma indicating hemosiderin deposits (arrow). (E) Vascular disruption in the subarachnoid space with proliferation of meningeal cells. $(F)$ Higher power to show the hyalinized subendothelial matrix $(*)$ and meningeal cell proliferation. Scale bars: $A, B, D$, and $E=250 \mu \mathrm{m} ; C$ and $F=40 \mu \mathrm{m}$.

in either ventilated group compared with controls $(p>0.05)$. There was an increase $(p<0.05)$ in the density of astrocytes in the hippocampus in PPV animals (Fig. 3E) but not in PPV + iNO animals (Fig. $3 F$ ) compared with controls (Fig. 3D).

Areal density of oligodendrocytes (Table 3). There was a tendency $(p<0.09)$ for a decrease in the areal density of MBP-positive oligodendrocytes in PPV (137.2 \pm 8.8 cells/ $\left.\mathrm{mm}^{2}\right)$ and PPV $+\mathrm{iNO}\left(120.6 \pm 24.3\right.$ cells $\left./ \mathrm{mm}^{2}\right)$ animals compared with controls $\left(190.0 \pm 24.3\right.$ cells $\left./ \mathrm{mm}^{2}\right)$.

Percentage of white matter occupied by blood vessels. This was not significantly different between groups $(p>$ 0.05 ) in either the subcortical (control, $1.4 \pm 0.2 \%$; PPV, $1.9 \pm 0.3 \%$; PPV + iNO $2.2 \pm 0.3 \%$ ) or deep white matter (control, $1.2 \pm 0.1 \%$; PPV, $1.8 \pm 0.5 \%$; PPV + iNO $1.8 \pm$ $0.3 \%)$ regions.

Semiquantitative assessment-Radial glia. Intensely GFAP-IR fibers were present at the ventricular surface projecting into the deep white matter in all control animals. Fibers were present in the majority of animals in the ventilated groups (Table 3). Although there was no difference in scores between groups $(p>0.05)$, there was a tendency for the PPV animals to have fewer fibers.

Perivascular cuffing in the deep white matter was evident in all animals; there was no difference between groups.
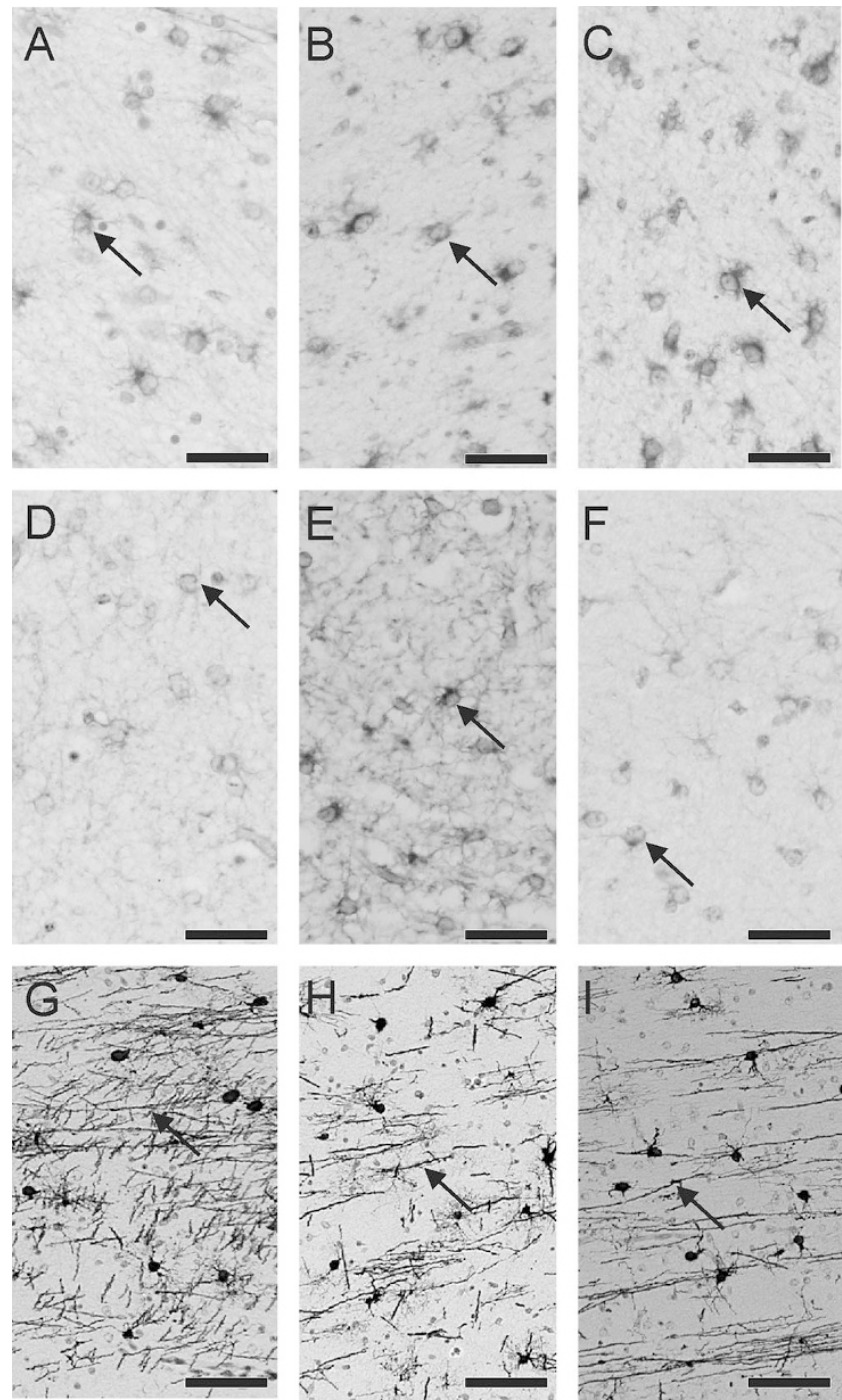

Figure 3. $(A-C)$ GFAP-IR in the deep white matter $(A)$ Gestational control, arrows indicate astrocytes; $(B) \mathrm{PPV}$; (C); PPV + iNO. Astrocyte density was increased in PPV + iNO compared with PPV and control animals. $(D-F)$ GFAP-IR sections in the hippocampus. (D) Gestational control, arrows indicate astrocytes. (E) PPV. (F); PPV + iNO. Astrocyte numbers were increased in PPV compared with PPV + iNO and control animals. $(G-I)$ Myelin basic protein-IR sections. $(G)$ control. (H) PPV. (I) PPV + iNO. Arrows indicate myelinated fibers. $(H)$ Reduced myelination in PPV and PPV + iNO animals compared with controls. $(A-F)=125 \mu \mathrm{m} ;(G-I)=100 \mu \mathrm{m}$.

Myelination. In controls, myelination was present in the thalamus and posterior limb of the internal capsule (Fig. 3G); immunoreactive fibers were also observed in the hippocampus and white matter of the pre- and postcentral gyri. Semiquantitative assessment revealed a reduction in myelination in both PPV $(p<0.01$; Fig. $3 H)$ and PPV + iNO $(p<0.05$; Fig. $3 I)$ animals compared with controls (Table 3 ).

Brain damage index (Table 3). There was an increase ( $p<$ $0.001)$ in the brain index score for both ventilated groups compared with controls (Fig. 1B); there was no difference $(p>$ $0.05)$ between ventilated groups.

Relationship of brain injury to brain volume and physiology. Physiologic responses in these animals has been described previously (16). There was no difference $(p>0.05)$ in $\mathrm{pH}$, $\mathrm{FiO}_{2}, \mathrm{PaCO}_{2}$ or MAP minimum and maximum or flux (maxi- 


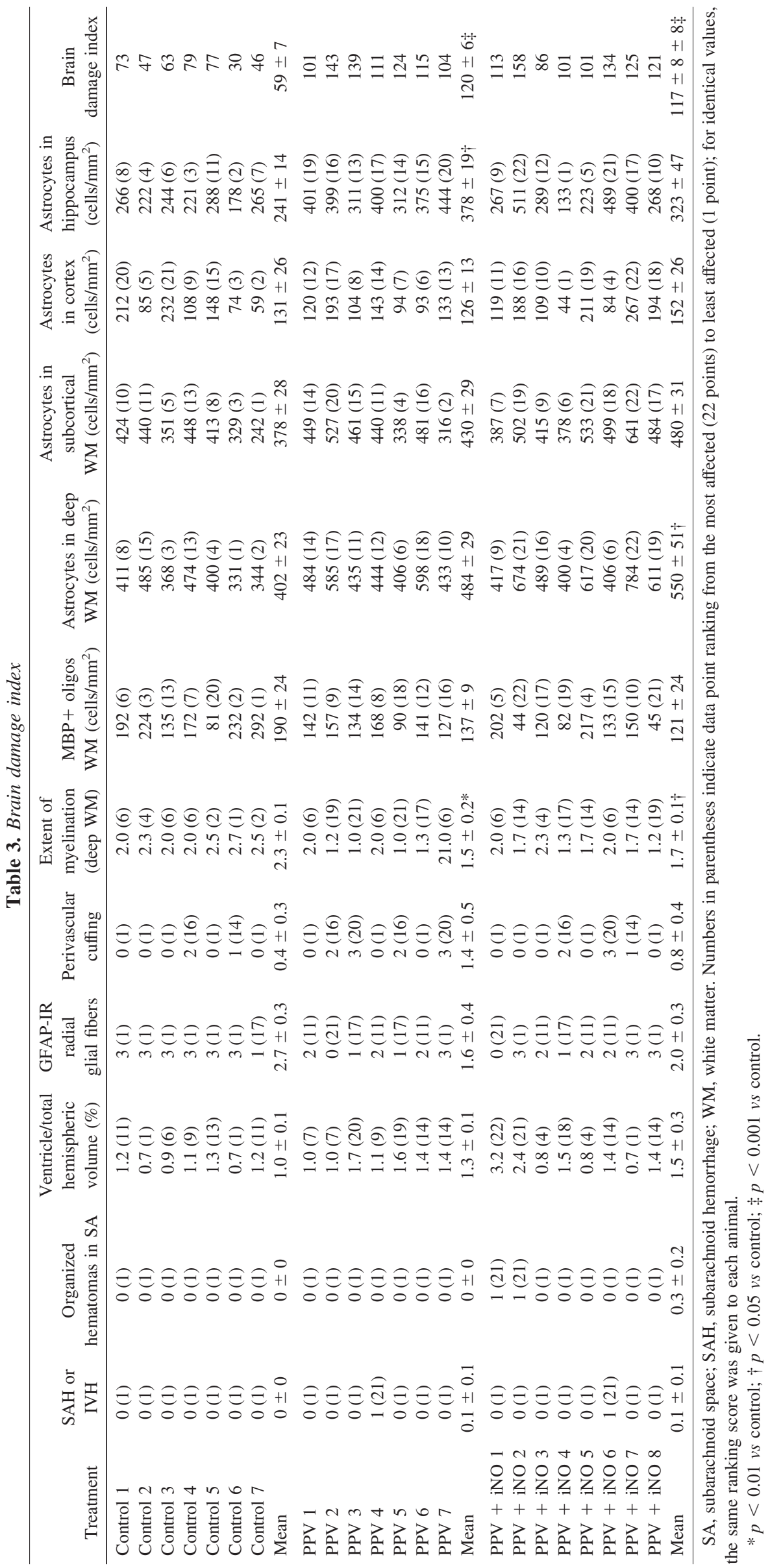


mum, median, or average) values between PPV and PPV + iNO treated animals. There was no correlation between any physiologic parameter and the brain damage $(p>0.05)$ or brain growth indices $(p>0.05)$, white matter volume and the density of white matter astrocytes $(p>0.05)$, the extent of myelination and brain size or ventricular volumes $(p>0.05)$.

\section{DISCUSSION}

Baboon infants prematurely delivered at $67 \%$ of gestation and treated by PPV with or without iNO for approximately $14 \mathrm{~d}$ exhibited delayed body and brain growth with subtle cerebral neuropathologies compared with gestational controls. These alterations occurred in the absence of potentiating factors such as perinatal infection or hypoxia. There was no evidence that iNO treatment resulted in a major shift of either risk or benefit in injury and/or development as there was no difference in the growth and development or brain damage indices between treatment groups. However, iNO therapy resulted in a small increase in subarachnoid hemorrhages (SAH) and increased deep white matter astrocytosis, although there was a trend toward an improvement in the SFI. We acknowledge that there are limitations in our study due to the small number of animals available for study and to the equal weighting given to all neuropathologies in the brain damage index. At this stage, it is difficult to determine which neuropathologies might be the most relevant predictors of any long-lasting deficits. Longer exposure to iNO or longer survival times after the current exposure regimen would be instructive in clarifying the outcomes associated with this therapy, but such protocols were not feasible in the present study.

There was brain sparing in both cohorts of ventilated animals as evidenced by an increased brain-to-body weight ratio. However, in absolute terms, there was a reduction in all volumetric measurements in the cerebral hemispheres compared with gestational controls; there was no difference between ventilated groups in any of these measures. The major site of pathology in the brains overall was the cerebral white matter as has been previously described in this model (15). Semiquantitative assessment indicated a delay in the extent of myelination in both groups; this did not appear to be influenced by the presence or absence of iNO. The delay correlated with a tendency for MBP-positive oligodendrocytes to be reduced in number due to either a delay in maturation of the lineage or to oligodendrocyte death as a result of oxidative and/or nitrosative stress $(20,21)$. In addition, the reduction in myelin formation could be due to reduced axonal numbers and/or to reduced axonal diameter and hence thinner myelin sheaths.

Astrogliosis also occurred to some extent in both ventilated groups compared with gestational controls; there were significant increases in astrocyte density in the deep white matter with iNO therapy and in the hippocampus without therapy. Reactive gliosis likely occurs as a response to oxidative stress as a result of cerebral hypoxemia (22). Despite attempts to maintain respiratory stability, hypoxic episodes occurred in the baboon model as they do in the preterm human infant.
A notable finding was that organizing hematomas in the subarachnoid space were only associated with iNO therapy. $\mathrm{SAH}$ and IVH have been more uncommon in the premature baboon than in the human preterm infant (15), and we have not observed this extent of subarachnoid bleeding in any other series of models studied to date. The occurrence of this neuropathology might result in ongoing damage as it was associated with evidence of recent hemorrhage and hence with the potential release of iron, which could catalyze free radicalmediated brain injury. The potential for further subarachnoid fibrosis during the repair process and subsequent disruption to normal CSF circulation can be postulated if hematomas were ongoing and persistent. Indeed, the two animals with hematomas were also the animals with ventriculomegaly. No incidence of IVH was observed in any animal in any group. Thus, we conclude that iNO appeared to exacerbate disruption of the subarachnoid vasculature in some animals but did not result in an increased risk of IVH. There were no specific physiologic correlations that allow us to propose why particular animals were vulnerable to vascular damage. At this stage, we cannot explain why damage occurred in subarachnoid but not intraparenchymal vessels.

Gyrification of the cerebral hemispheres is an important index of brain development and is thought to reflect development of cerebral and subcortical connectivity (23). Here we have shown that, although brain weight was reduced in both ventilated groups compared with controls, overall gyrification was delayed in PPV animals but not in the cohort with iNO therapy. In PPV animals, reductions were observed in all lobes except in the later developing frontal region. Our results suggest that iNO may confer a beneficial effect on the development of cortical gyrification. It is unlikely that this effect would occur via white matter protection as white matter volumes were reduced in both cohorts, and indeed deep white matter astrogliosis was marginally increased in the iNOtreated animals. Thus, the impact of iNO in protecting cortical development may be mediated by a direct neuronal effect. The application of advanced MRI may better delineate brain surface morphology and gyrification in this model (24); these techniques are now being used in preterm infants $(25,26)$ and could be applied to recent randomized trials of iNO in human preterm infants.

In conclusion, we have shown that subtle neuropathologic alterations are evident in all animals delivered prematurely and maintained with neonatal intensive care for $14 \mathrm{~d}$, compared with gestational controls. Overall, iNO did not alter the extent of the damage within the cerebral parenchyma. These results do not confirm any protective effects or major injurious effects of nitric oxide therapy on the developing brain, although they are consistent with the National Institute of Child Health and Development study that hemorrhagic risk may be increased and yet there may be effects on gray matter and gyral development that may be independent.

Acknowledgments. The authors are most grateful to Ms. Winter and Dr. Coalson at the SFBR for provision of baboon tissue and to Drs. Folkerth and Kinney, Department of Pathology, Children's Hospital, Boston, MA, for neuropathologic 
advice. Dr. Dalitz provided invaluable assistance with some of the immunohistochemistry.

\section{REFERENCES}

1. Hack M, Taylor HG 2000 Perinatal brain injury in preterm infants and later neurobehavioral function. JAMA 284:1973-1974

2. Wilson-Costello D, Friedman H, Minich N, Fanaroff AA, Hack M 2005 Improved survival rates with increased neurodevelopmental disability for extremely low birth weight infants in the 1990s. Pediatrics 115:997-1003

3. Kinsella JP, Abman SH 2005 Inhaled nitric oxide therapy in children. Paediatr Respir Rev 6:190-198

4. Subhedar NV, Shaw NJ 1997 Changes in oxygenation and pulmonary haemodynamics in preterm infants treated with inhaled nitric oxide. Arch Dis Child Fetal Neonatal Ed 77:F191-F197

5. Kinsella JP, Cutter GR, Walsh WF, Gerstmann DR, Bose CL, Hart C, Sekar KC, Auten RL, Bhutani VK, Gerdes JS, George TN, Southgate WM, Carriedo H, Couser RJ, Mammel MC, Hall DC, Pappagallo M, Sardesai S, Strain JD, Baier M, Abman SH 2006 Early inhaled nitric oxide therapy in premature newborns with respiratory failure. N Engl J Med 355:354-364

6. Cannon RO 3rd, Schechter AN, Panza JA, Ognibene FP, Pease-Fye ME, Waclawiw MA, Shelhamer JH, Gladwin MT 2001 Effects of inhaled nitric oxide on regional blood flow are consistent with intravascular nitric oxide delivery. J Clin Invest 108:279-287

7. Schreiber MD, Gin-Mestan K, Marks JD, Huo D, Lee G, Srisuparp P 2003 Inhaled nitric oxide in premature infants with the respiratory distress syndrome. $\mathrm{N}$ Engl J Med 349:2099-2107

8. Van Meurs KP, Wright LL, Ehrenkranz RA, Lemons JA, Ball MB, Poole WK, Perritt R, Higgins RD, Oh W, Hudak ML, Laptook AR, Shankaran S, Finer NN, Carlo WA, Kennedy KA, Fridriksson JH, Steinhorn RH, Sokol GM, Konduri GG, Aschner JL, Stoll BJ, D’Angio CT, Stevenson DK 2005 Inhaled nitric oxide for premature infants with severe respiratory failure. N Engl J Med 353:13-22

9. Subhedar NV, Ryan SW, Shaw NJ 1997 Open randomised controlled trial of inhaled nitric oxide and early dexamethasone in high risk preterm infants. Arch Dis Child Fetal Neonatal Ed 77:F185-F190

10. Ballard RA, Truog WE, Cnaan A, Martin RJ, Ballard PL, Merrill JD, Walsh MC, Durand DJ, Mayock DE, Eichenwald EC, Null DR, Hudak ML, Puri AR, Golombek SG, Courtney SE, Stewart DL, Welty SE, Phibbs RH, Hibbs AM, Luan X, Wadlinger SR, Asselin JM, Coburn CE 2006 Inhaled nitric oxide in preterm infants undergoing mechanical ventilation. N Engl J Med 355:343-353

11. Bennett AJ, Shaw NJ, Gregg JE, Subhedar NV 2001 Neurodevelopmental outcome in high-risk preterm infants treated with inhaled nitric oxide. Acta Paediatr 90:573576
12. Mestan KK, Marks JD, Hecox K, Huo D, Schreiber MD 2005 Neurodevelopmental outcomes of premature infants treated with inhaled nitric oxide. $\mathrm{N}$ Engl J Med 353:23-32

13. Gidday JM, Shah AR, Maceren RG, Wang Q, Pelligrino DA, Holtzman DM, Park TS 1999 Nitric oxide mediates cerebral ischemic tolerance in a neonatal rat model of hypoxic preconditioning. J Cereb Blood Flow Metab 19:331-340

14. Peeters-Scholte C, Koster J, Veldhuis W, van den Tweel E, Zhu C, Kops N, Blomgren K, Bar D, van Buul-Offers S, Hagberg H, Nicolay K, van Bel F, Groenendaal F 2002 Neuroprotection by selective nitric oxide synthase inhibition at 24 hours after perinatal hypoxia-ischemia. Stroke 33:2304-2310

15. Dieni S, Inder T, Yoder B, Briscoe T, Camm E, Egan G, Denton D, Rees S 2004 The pattern of cerebral injury in a primate model of preterm birth and neonatal intensive care. J Neuropathol Exp Neurol 63:1297-1309

16. McCurnin DC, Pierce RA, Chang LY, Gibson LL, Osborne-Lawrence S, Yoder BA, Kerecman JD, Albertine KH, Winter VT, Coalson JJ, Crapo JD, Grubb PH, Shau PW 2005 Inhaled NO improves early pulmonary function and modifies lung growth and elastin deposition in a baboon model of neonatal chronic lung disease. Am J Physiol Lung Cell Mol Physiol 288:L450-L459

17. Loeliger M, Inder T, Cain S, Ramesh RC, Camm E, Thomson MA, Coalson J, Rees S 2006 Cerebral outcomes in a preterm baboon model of early versus delayed nasal continuous positive airway pressure. Pediatrics 118:1640-1653

18. Rees S, Stringer M, Just Y, Hooper SB, Harding R 1997 The vulnerability of the fetal sheep brain to hypoxemia at mid-gestation. Brain Res Dev Brain Res 103:103118

19. Duncan JR, Cock ML, Harding R, Rees SM 2000 Relation between damage to the placenta and the fetal brain after late-gestation placental embolization and fetal growth restriction in sheep. Am J Obstet Gynecol 183:1013-1022

20. Haynes RL, Folkerth RD, Keefe RJ, Sung I, Swzeda LI, Rosenberg PA, Volpe JJ, Kinney HC 2003 Nitrosative and oxidative injury to premyelinating oligodendrocytes in periventricular leukomalacia. J Neuropathol Exp Neurol 62:441-450

21. Back SA, Han BH, Luo NL, Chricton CA, Xanthoudakis S, Tam J, Arvin KL, Holtzman DM 2002 Selective vulnerability of late oligodendrocyte progenitors to hypoxia-ischemia. J Neurosci 22:455-463

22. Cai Z, Lin S, Fan LW, Pang Y, Rhodes PG 2006 Minocycline alleviates hypoxicischemic injury to developing oligodendrocytes in the neonatal rat brain. Neuroscience 137:425-435

23. Van Essen DC 1997 A tension-based theory of morphogenesis and compact wiring in the central nervous system. Nature 385:313-318

24. Kroenke CD, Bretthorst GL, Inder TE, Neil JJ 2005 Diffusion MR imaging characteristics of the developing primate brain. Neuroimage 25:1205-1213

25. Inder TE, Warfield SK, Wang H, Huppi PS, Volpe JJ 2005 Abnormal cerebral structure is present at term in premature infants. Pediatrics 115:286-294

26. Deipolyi AR, Mukherjee P, Gill K, Henry RG, Partridge SC, Veeraraghavan S, Jin H, Lu Y, Miller SP, Ferriero DM, Vigneron DB, Barkovich AJ 2005 Comparing microstructural and macrostructural development of the cerebra cortex in premature newborns: diffusion tensor imaging versus cortical gyration. Neuroimage 27:579-586 